\title{
Analogues of Cores and Stars in Simulated Molecular Clouds
}

\author{
James Wadsley ${ }^{1}$, Michael Reid ${ }^{2}$, Farid Qamar ${ }^{1}$, Alison Sills ${ }^{1}$ and \\ Nicholas Petitclerc ${ }^{1}$ \\ ${ }^{1}$ Dept. Physics \& Astronomy, McMaster University, 1280 Main St. W., Hamilton, ON, L8S \\ 4M1, Canada \\ corresponding author email: wadsley@mcmaster.ca \\ ${ }^{2}$ Dept. Astronomy \& Astrophysics, University of Toronto, ON, M5S 3H4, Canada
}

\begin{abstract}
We use images derived from collapsing, turbulent molecular cloud simulations without sinks to explore the effects of finite image angular resolution and noise on the derived clump mass function. These effects randomly perturb the clump masses, producing a lognormal clump mass function with a Salpeter-like high mass end. We show that the characteristic break mass of the simulated clump mass functions changes with the angular resolution of the images in a way that is entirely consistent with the observations. We also present some cautionary tales regarding sink particles and highlight the need to ensure that sinks actually correspond to distinct collapsing objects. We test several popular numerical sink criteria in the literature and compare to converged, non-sink results.
\end{abstract}

Keywords. ISM: structure, submillimeter, methods: data analysis, stars: mass function, stars: formation, methods: numerical

\section{Clump Mass Functions}

The properties of molecular cloud clumps are increasingly being used as a tool to test theories of star formation. Recent measurements of the shape of the clump mass function $(\mathrm{CMF})$ in nearby low-mass star-forming regions appear to agree with the shape of the stellar initial mass function (Motte et al. 2001; Johnstone et al. 2001; Tothill et al. 2002). The different peak masses are taken to be indicative of the star formation efficiency. However, Reid \& Wilson (2006) have shown that the interpretation of observational clump mass functions is biased by the effects of small-number statistics and certain fitting techniques.

Maps of the dust continuum emission in star-forming regions are sectioned into clumps either by eye or using one of a variety of algorithms, such as clfind2d (Williams, de Geus, \& Blitz 1994). Clfind2d has been criticized as inaccurate (Pineda, Rosolowsky, \& Goodman 2009;Curtis \& Richer 2010) but it remains popular and the effects investigated here affect all methods. We used clfind2d to identify clumps in the simulated image in the left panel of fig. 1. The cloud was simulated with the GASOLINE (Wadsley, Stadel \& Quinn, 2004) SPH code and contains 5000 solar masses (see Reid et al. 2010, Petitclerc 2009). We applied different beams, equivalent to placing the cloud at distances of $160 \mathrm{pc}$ ( $\rho$-Ophiuchus), $450 \mathrm{pc}$ (Orion), $1 \mathrm{kpc}$ and $2 \mathrm{kpc}$. We also added noise approximating a 10'x10' SCUBA $850 \mu \mathrm{m}$ scan map over 10 hours in grade 2 weather (roughly $0.03 \mathrm{Jy}$ beam $^{-1}$ ) and progressively worse noise by factors of two. The number of objects found changes at all masses. Thus there is no equivalent of a completeness limit as for point sources. The perturbing effects of changing beam and noise generate a log-normal CMF in all cases (c.f. Larson 1973). Furthermore, the high mass end is well-fit by Salpeter-like 

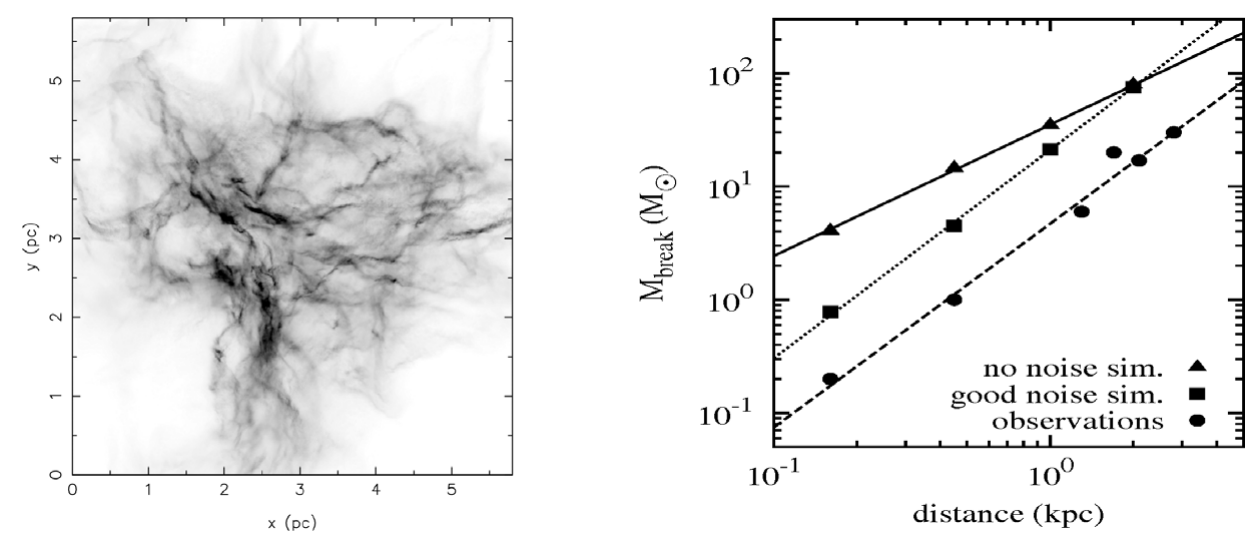

Figure 1. Left panel - simulated observation (noise-free). Right Panel - Break mass versus distance to various observed and simulated star-forming regions. Break masses from the observational data are shown with filled circles, while those from the simulations with noise-free and good noise are shown with filled triangles and squares, respectively. The lines of best fit shown are power laws in distance with exponents of 1.2 for the noise-free simulations (solid line) and 1.8 for both the simulations with good noise (dotted line) and the observations (dashed line).

power-law. Simulated images, mass functions and fits for all cases are presented in Reid et al. (2010).

The high mass end for each case was fit with a double power-law intersecting at a characteristic break mass. The break moves to higher masses for more distant objects with a fixed beam (i.e. poorer angular resolution). This behavior tracks that seen for observations of progressively more distant star forming regions, as shown in the right panel of fig. 1.

We conclude that CMF measurements to date have not provided unambiguous measurements of either the intrinsic shape or the characteristic mass scales of the clump mass function due to the effects of noise and resolution. This situation may be about to change, thanks to upcoming observations to be made with instruments such as the Submillimetre Common-User Bolometer Array 2 (SCUBA2) on the James Clerk Maxwell Telescope (JCMT) as well as the Spectral and Photographic Imaging Receiver (SPIRE) and the Photodetector Array Camera and Spectrometer (PACS) on the Herschel Space Observatory.

\section{Sink Particles}

Sink particles are a numerical technique that allows simulators to model individual star formation over the time-scale of an entire molecular cloud collapse: to go from the CMF to the IMF. The basic premise behind sink particles is that strongly bound regions arise in self-gravitating flows where material becomes permanently locked up. In the case of star formation, neglecting stellar outflows (which may be treated separately), this is probably a reasonable assumption near a proto-star. If inflows are supersonic there is little hydrodynamic back reaction from the accreted material to the surroundings. A region including each protostar is replaced with a sink with the same mass and a reasonable boundary condition for the gas dynamics. Sinks have been used in star formation simulations for some time (e.g. Bate, Bonnell \& Price 1995) and have been implemented in many different codes and forms.

As sink formation is irreversible, it is important to introduce sinks only where individual collapse is inevitable. In all sink approaches, spherical candidate sink regions are 

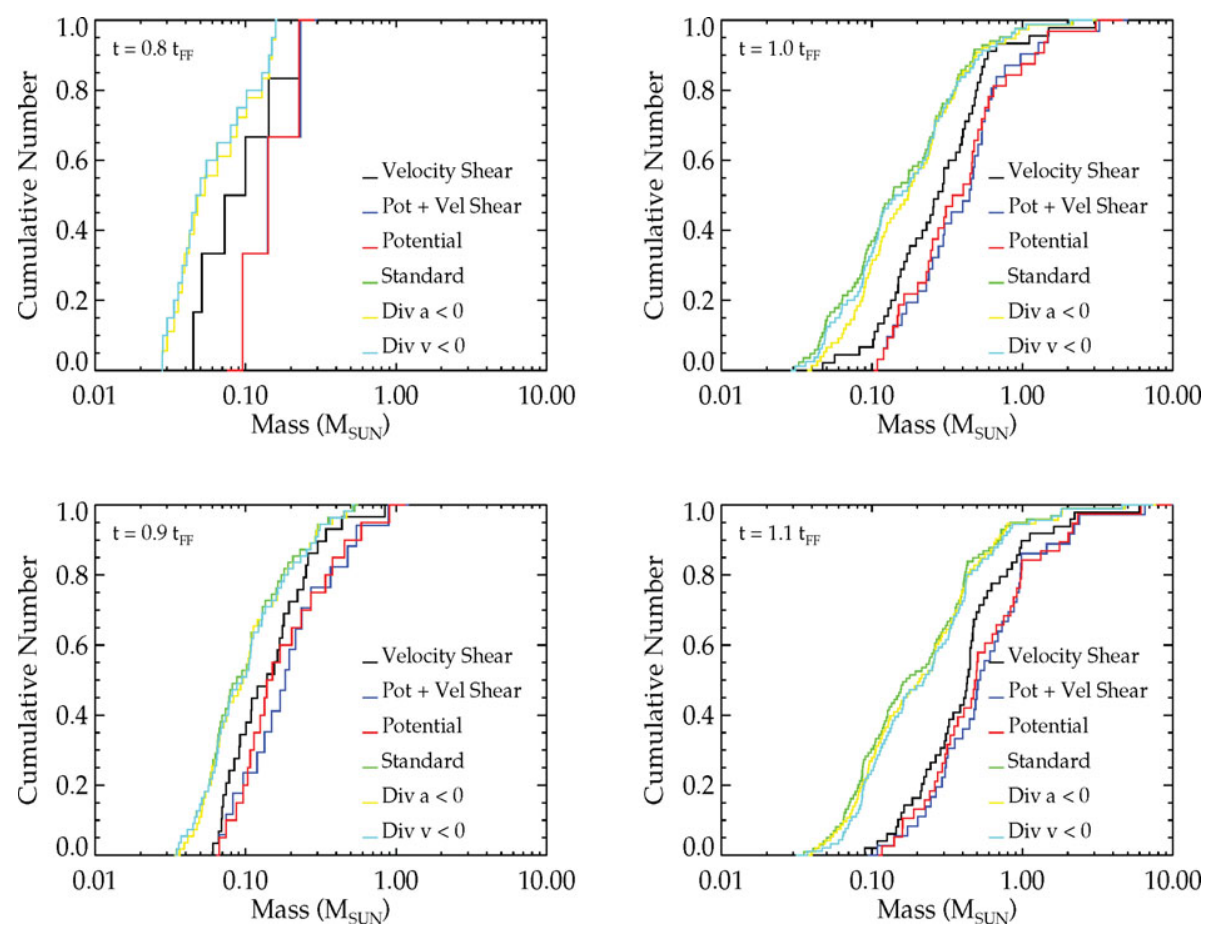

Figure 2. Cumulative mass function of sink particles with different sink formation criteria. Potential and/or velocity shear based criteria are necessary to avoid spurious low mass sinks.

identified when the local gas density exceeds either a fixed value (e.g. Bate 1995) or a value tied to the local Jeans criterion (e.g. Krumholz et al. 2004). Additional criteria have been introduced to ensure that the region is collapsing individually, e.g.:

(a) Bound: $E_{\text {Total }}<0$

(b) Rotationally Bound: $E_{\text {rotational }}+E_{\text {Thermal }}+E_{\text {Gravity }}<0$

(c) Virialized (Thermally): $E_{\text {Thermal }}<\frac{1}{2}\left|E_{\text {Gravity }}\right|$

(d) Flow is accelerating: $\nabla \cdot \mathbf{a}<0$, i.e. $d^{2} \log \rho / d t^{2}>0$

(e) Density $(\rho)$ maximum

(f) Potential $(\phi)$ minimum $(\mathrm{NEW})$

(g) Flow is converging: $\nabla \cdot \mathbf{v}<0$, i.e. $d \log \rho / d t>0$

(h) Flow is converging in all directions: eigenvalues of $d v_{i} / d x_{j}$ negative (NEW)

Bate, Bonnell \& Price (1995) (SPH) implemented criteria (a-d). (a) implies (b) so test (b) will not be considered further. Test (e) tends to occur naturally as sink candidates first reach the threshold density. Krumholz et al. 2004 (AMR) performed no explicit tests but the use of a Jeans criterion for the threshold density is equivalent to test (a) for boundness. They also aggressively merge sinks to mop up excess sink creation though no merging is considered here. Attwood et al. 2009 (SPH) used test (a) and flow convergence (g). Federrath et al. 2010 tested various criteria on a 100 solar mass turbulent cloud collapse, using the FLASH AMR code (Fryxell et al. 2000), trying standard: bound density maxima (a,c,e) and new approaches: bound potential minima with flows converging in all directions (a,c,f,h). A key result was that standard approaches create extra sinks in filaments. There are clear circumstances when bound gas cannot be assumed to be forming new collapsed object: 
1) Bound structures such as filaments fragment slowly via well defined hydrodynamic processes (Larson 1985). Preemptively chopping the filament into sinks imposes an artificial numerical mass scale for the proto-stars (see Figure 14 of Federrath et al. 2010).

2) Material infalling onto deep potentials well may be intrinsically bound when considered in isolation but velocity shear along the infall direction inhibits local collapse so that the gas should accrete onto the centre without fragmenting.

Federrath et al. 2010 re-ran their initial conditions using the Bate, Bonnell \& Price (1995) code and sink conditions (a-d) and saw similar sink numbers to their new criteria. However, the gravity resolution was much lower in the SPH runs, similar to the sink radii $\left(r_{S I N K}=500 A U\right)($ private communication). We re-ran the same initial condition using the GASOLINE SPH code with gravitational resolution an order of magnitude better, similar to the FLASH runs, and found excessive filament fragmentation in SPH similar to the right column of their figure 14. Thus for the same sink criteria and resolution SPH and AMR do agree that boundness and related criteria (i.e. a-c) are insufficient. Our cumulative sink mass functions are shown in figure 2. The results fall into two, clearly separated categories (e.g. by K-S tests), particularly at later times. Using simple boundness or tests on the mass accumulation $(\mathrm{d}, \mathrm{g})$, we find that roughly twice as many low mass sinks are generated, particularly in filaments. Numerically, tests such as $\nabla \cdot \mathbf{v}<0$ are ill-posed because noise in the velocity field can make the measure fluctuate about zero so that sink candidates are likely to pass if they are tested a few times.

We find that the new sink criteria proposed by Federrath et al. 2010, namely using potential minima (f) or velocity shear (h), are more robust. Our results are consistent with theirs, as presented in their figure 16. However, the authors did not demonstrate that this is the correct result. To do so, we ran the collapse without sinks to much higher densities at the same mass resolution and again with 8 times higher mass resolution and confirmed that filaments form bound collapsing objects only within $\sim 500 \mathrm{AU}$ of the sinks allowed by the new criteria.

\section{References}

Attwood, R., Goodwin, S., Stamatellos, D. \& Whitworth, A. 2009, A\&A A, 495, 201

Bate, M., Bonnell, I. \& Price, N. 1995, MNRAS, 277, 362

Curtis, E. I. \& Richer, J. S. 2010, MNRAS, 402, 603

Federrath, C., Banerjee, R., Clark, P. \& Klessen, R. 2010, ApJ, 713, 269

Fryxell et al. 2000, ApJS, 131, 273

Johnston, D. et al. 2001, ApJ, 559, 307

Kratter, K., Matzner, C., Krumholz, M., \& Klein, R. 2010, ApJ, 708, .1585

Krumholz, M., McKee, C., Klein, R. 2004, ApJ, 611, 399

Larson, R. B. 1973, MNRAS, 161, 133

Larson, R. B., 1985, MNRAS, 214, 379

Motte, F., et al. 2001, A\&SA, 372, L41

Petitclerc, N. 2009, Ph.D. Thesis, McMaster University

Pineda, J. E., Rosolowsky, E. W., \& Goodman, A. A. 2009, ApJ, 699, L134

Reid, M., Wadsley, J., Petitclerc, N., \& Sills, A. 2010, ApJ, accepted

Reid, M. A. \& Wilson, C. D. 2006, ApJ, 650, 970

Tothill et al. 2002, ApJ, 580, 285

Wadsley, J. W., Stadel, J., \& Quinn, T. 2004, New Astronomy, 9, 137

Williams, J. P., de Geus, E. J., \& Blitz, L. 1994, ApJ, 428, 693 